\title{
The
}

\section{Internet et enseignants : entre contrastes et clivages. Enquête auprès d'enseignants du secondaire}

Caroline LADAGE, Jean RAVESTEIN (Aix Marseille Université, ADEF EA 4671, ENS Lyon, IFE, 13248, Marseille, France)

RÉSUMÉ • Notre travail à visée compréhensive questionne ce que déclarent faire les enseignants du secondaire avec les TIC, en focalisant sur l'usage d'Internet, ainsi que ce qu'ils croient que font leurs élèves avec cet instrument. L'objectif est de mieux cerner la grammaire des pratiques de l'Internet dans le champ du didactique. On découvre chez les 2862 enseignants interrogés que des variables (discipline, genre, âge, ...) jouent sur les usages des outils numériques et sur la place qu'ils leur accordent dans leurs pratiques professionnelles. Notre enquête révèle des positions contrastées, des clivages d'opinion qui témoignent d'un manque de maturité épistémologique actuel. Les résultats dessinent aussi les contours de l'évolution des mentalités enseignantes qui laissent augurer qu'un avenir prometteur est possible pour les usages d'Internet en éducation et en formation alors qu'il demeure aujourd'hui encore insuffisamment raisonné.

MOTS-CLÉS • utilisation de l'informatique, Internet, didactique, enseignement secondaire, stéréotypes

DONNÉES ASSOCIÉES • Accessibles via le lien permanent (Datapublication.org : Tge-adonis.fr.) http://hdl.datapublication.org/11107/p-001-017

ABSTRACT • Our comprehensive work questions secondary teachers' statements on their ICT usages, in particular Internet, as well as what they believe students do with it. The goal is to better understand the use of Internet in education. We discover that among the 2862 teachers we interviewed, variables (discipline, gender, age...) influence their use of digital tools and the place they give them in their professional practices. Our investigation reveals contrasting positions, divisions of opinion that reflect a lack of epistemological maturity today. The results also draw the outlines of the evolution of teachers' mentalities that augur a promising future is possible for the use of Internet in education and training while today it is still insufficiently reasoned.

KEYWORDS - computer usage, Internet, didactics, secondary education, stereotypes

ASSOCIATED DATA: http://hdl.datapublication.org/11107/p-001-017 


\section{Introduction}

De nombreux rapports et projets témoignent de la vigueur des démarches institutionnelles pour intégrer les TIC dans le système éducatif français ${ }^{1}$. Les argumentaires se déploient selon deux axes principaux: l'instrumentation des situations d'apprentissage et une préparation à l'usage de l'informatique et de l'Internet au service du «devenir citoyen ». On ne peut laisser sans réponse la question de l'usage déclaré de ces outils par les enseignants. Le plan de développement de l'usage du numérique à l'école (2010) témoigne également de la vigilance de l'institution concernant les perspectives de rapports renouvelés - modernisés - aux savoirs savants. Les réformes instaurant les $\mathrm{B} 2 \mathrm{i}$ et les $\mathrm{C} 2 \mathrm{i}$, ainsi que le $\mathrm{C} 2 \mathrm{i}$ enseignant sont loin d'être remises en question dans la loi pour la refondation de l'école ${ }^{2}$, bien au contraire. À ce propos on peut se référer au numéro spécial Des usages des TIC à la certification des compétences numériques : quels processus de formation et de validation? coordonné par Cathia Papi et on peut repérer que ce type de préoccupation dépasse les frontières (Chen, 2010 ; Lei, 2009).

Avant même que l'accès à l'Internet haut débit soit devenu quasi généralisé dans les sociétés développées, les chercheurs en éducation ont pu montrer que cela transformait de manière générale les rapports aux savoirs et à leur enseignement, tant du côté des enseignants que des élèves (Selwyn, 1999 ; Becker, 1999). Dans certains cas Internet est vécu comme un élément perturbateur dans la transmission des connaissances (Anderson et Reed, 1998). D'autres travaux insistent sur les éléments facilitateurs pour l'enseignement (Ertmer et Ottenbreit-Leftwich, 2010). Accompagnant le haut débit et sa puissance, des études ont porté sur de nouvelles relations, parfois tendues ou même conflictuelles, entre le monde scolaire et l'Internet du fait de l'usage des ressources rendues très facile d'accès (Dawes, 2001 ; Hennessy, Ruthven et Brindley, 2005 ; Bielefeldt, 2006). Nombre d'entre elles ont insisté sur les résistances, obstacles, freins à l'usage des TIC dans le travail scolaire en particulier de la part des enseignants (Pelgrum, 2001 ; Schoepp, 2005 ; Gillespie, 2006 ; Afshari, Abu Bakar, Su Luan, Abu Samah et Say Fooi, 2009). Des explications nombreuses ont été avancées pour éclaircir cette question à travers la publication de rapports ou d'études d'envergure qui pointent à la fois les problèmes de moyens techniques manquants, de formation insuffisante (Balanskat, Blamire et Kefala, 2006 ; Osborne et Hennessy, 2003), voire de problèmes plus psychologiques chez les enseignants, concernant leurs rapports avec les techniques innovantes (Phelps, Graham et Kerr, 2004).

En France, la question de l'usage réel des TIC à titre professionnel par les enseignants a été souvent examinée dans le contexte de la mise en place de formations en ligne, surtout dans le supérieur, ainsi que sous l'angle de la nécessité de formation (C2i «enseignant») suite à la mise en place des B2i (rapport IGEN, 2001). Par 
contre, les prescriptions, les préconisations, les injonctions concernant l'usage des TIC par les enseignants font florès dès les années 2000 (DEP, 2003), contrastant avec un déficit d'analyse de situations concrètes de travail et des modalités selon lesquelles ils les affrontent (Tardif et Lessard, 1999). Pourtant, des chercheurs ont fait porter leurs efforts sur les dispositifs et outils favorisant l'appropriation des TIC par les enseignants (Blondel et Tort, 2007 ; Gueudet, Soury-Lavergne et Trouche, 2008, par exemple) et ont tracé des perspectives ou énoncé des mises en garde (Baron et Bruillard, 2002 ; Ravestein, Ladage et Johsua, 2007).

Ce travail questionne les rapports que les enseignants déclarent entretenir avec les TIC, grâce à un questionnaire en ligne renseigné par 2862 d'entre eux. L'objectif est de dresser un état de ces savoir-faire et de recueillir un nombre important de points de vue représentant aussi bien que possible ceux de cette population en France. Un attention particulière sera portée sur la question des effets de l'accès aux ressources que l'Internet permet dans et hors le cadre scolaire.

\section{Quels usages de l'informatique et d'Internet?}

La diffusion massive des TIC partout (portabilité), pour presque tous (coûts en baisse) et à tout moment (connectivité) est incontestablement un phénomène qui pose la question de leur usage scolaire (pratiques instrumentées qui se trouveraient facilitées) ainsi que celle, non moins importante, de la redéfinition de la mission de l'école dans le développement du numérique dans la société (MEN, 2008). Sans pouvoir apporter des éléments de réponse ici, cette diffusion inédite pose une double question à notre sens primordiale : quels savoirs savants pourraient justifier de bonnes pratiques à enseigner (questions didactiques de transposition et d'usage) et, partant de là, comment armer des dispositifs de formation efficaces pour tous les acteurs du système scolaire (question politique, que pointent Stiegler, Meirieu et Kambouchner, 2012) ? Si l'identification de pratiques intégrant efficacement les TIC à l'école est bien le fil rouge des différentes études que nous venons de citer, reste entier le problème de la multiplicité et de l'hétérogénéité des domaines d'usage des TIC. En effet, elles sont étudiées tantôt sous l'angle des pratiques des élèves, tantôt sous celui des pratiques enseignantes. On peut toutefois s'inquiéter de la difficile rencontre de ces deux mondes de pratiques (élèves et enseignants). À l'intérieur même de chacun, l'opposition entre pratiques professionnelles et personnelles semble déjà un premier obstacle difficile à surmonter.

Ainsi, côté élèves, Fluckiger et Bruillard (2008) observent un hiatus, voire une opposition forte, entre pratiques personnelles et pratiques scolaires, qui expliquerait l'intégration difficile des usages des TIC à l'école. Ces auteurs mettent en évidence que les compétences en TIC développées par les élèves dans leurs univers personnels s'avèrent faiblement mobilisées en contexte scolaire. Ils l'expliquent par un niveau de 


\section{Caroline LADAGE, Jean RAVESTEIN}

connaissances techniques finalement limité et par une différence de contexte d'usage : « Nous défendons l'idée que ces transferts entre des univers d'appropriation distincts sont loin d'être aussi aisés que certaines similarités de surface (dans les outils utilisés ou la manière de les employer) pourraient le laisser penser, et que les compétences acquises sont bien davantage locales et contextualisées que transversales. » (Fluckiger et Bruillard, 2008)

Ce constat d'une structuration différente de l'activité qui fait obstacle au réinvestissement scolaire des compétences techniques des élèves est confirmé par Gueudet, Soury-Lavergne et Trouche (2008).

Toujours du côté des élèves, la tentative de croisement entre les régimes temporels des lycéens et les significations d'usage d'Internet de Le Douarin et DelaunayTéterel (2011) montre que «L'organisation fine du temps libre et des activités se dérobe aux évidences (...) Chacun, dans le régime temporel qui est le sien, favorise tantôt des pratiques d'exercisation, tantôt des modes de renforcement des savoirs ou encore des activités de compensation pour vérifier la qualité des enseignements et les compléter si nécessaire ». On voit donc toute la difficulté pour les enseignants de cerner l'activité de leurs élèves avec Internet, question que nous tenterons d'élucider dans cette recherche. Toutefois pour être plus exhaustif on aurait pu se demander si l'enseignant a scénarisé dans son activité pédagogique l'utilisation des TIC ou pas et la mesure d'une telle scénarisation sur l'activité de ses élèves. Cette question pourrait faire l'objet d'une recherche complémentaire, ici nous y répondrons latéralement dans le repérage de besoins d'accompagnement et de formation dans les activités scolaires de l'Internet.

Côté enseignants, la commission Pochard dans son Livre vert sur l'état du métier enseignant (MEN, 2008a) désigne l'importance du poids de la forte identité professionnelle et de la conception marquée que les enseignants ont de leur métier, «forgée au cours de l'histoire de l'enseignement », sur la façon dont l'enseignement est dispensé. Selon le même rapport, la revendication de la «liberté pédagogique » pèse de plus en plus sur le fonctionnement du système éducatif. Cette revendication peut ralentir l'intégration et l'usage des TIC dans les pratiques pédagogiques. En effet, si l'instauration «d'une sorte de continuité entre le temps de la classe et le temps de travail des élèves en dehors de la classe » (MEN, 2008b) se généralise grâce aux TIC, cela peut être perçu comme une perte de «souveraineté ». La perméabilité accrue entre l'univers scolaire et l'univers privée peut être vu comme une désanctuarisation de l'école.

Un déficit de l'analyse de situations éducatives intégrant les TIC a été identifié par Bertrand (2004), qui n'en souligne pas moins la difficulté, tant cette intégration est marquée par l'influence de l'expérience personnelle et du rapport personnel de 
chaque enseignant à l'informatique et à l'Internet et souffre d'un manque d'intégration à un niveau collectif.

Le manque de diffusion des pratiques des TIC à l'école ne résulte pas tant d'un défaut d'intégration de pratiques personnelles de l'élève ou de celles de l'enseignant que d'une pénurie de pratiques et de savoirs de référence (Ladage, 2008). Ces savoirs sont aujourd'hui encore à identifier (et pour une part importante encore à construire), pour ensuite pouvoir étudier les conditions et contraintes de leur diffusion au sein de l'école et de la société.

Nous proposons de progresser dans cette argumentation en focalisant sur le champ qui nous occupe principalement dans ce travail, l'usage d'Internet, non pas dans toute la palette de ses possible, mais plus précisément en ce qui concerne l'accès à des ressources et aux stratégies de recherche d'informations afférentes.

Ladage (2008) note une péjoration culturelle de l'Internet qu'il y a lieu de dépasser en travaillant en amont sur une «étude précurriculaire », dont l'objectif principal serait d'enrichir le champ aujourd'hui encore très réduit des systèmes d'activités où sont confinées les pratiques de la recherche d'information sur Internet. L'étude du corpus de manuels et d'ouvrages disponibles sur le sujet témoigne d'un manque de pratiques élaborées et met en évidence que les usages que nous connaissons sont encore trop récents pour intégrer largement ce que l'on peut réellement faire sur et avec Internet (Ladage, 2008). Alors que naît à peine la genèse historique des pratiques de recherche d'information et des usages de l'Internet, nous vivons déjà actuellement un déni de la problématicité de ces pratiques, ce qui pourrait bien constituer un frein durable à leur évolution. Dans une étude menée par Ladage et Chevallard (2011), Internet apparaît en effet comme une réalité dénigrée, ce qui amène ces auteurs à noter, à propos d'une pédagogie de l'enquête encore balbutiante : « Il n'est sans doute pas facile d'utiliser l'Internet comme outil d'enquête lorsqu'on est plongé dans un univers qui regarde cet outil comme peu fiable, superficiel, irritant et froid. La chose est d'autant plus prégnante que, parce qu'on regarde l'Internet comme «facile », on est porté à ne pas en étudier les possibilités et à ne pas approfondir la connaissance des fonctionnalités qu'il offre. » Ces mêmes auteurs ont bien montré que la question de la fiabilité des ressources n'a plus lieu de se poser à partir du moment où chaque ressource peut être considérée comme un élément de réponse potentiel à un questionnement sans cesse renouvelé. À titre d'exemple les travaux sur la pédagogie de l'enquête citent fréquemment le recours efficace à Wikipédia dans le déroulement des enquêtes menées avec les élèves (Ladage et Chevallard, 2011).

Cette analyse amène ces auteurs à rappeler l'importance de renforcer le développement d'une culture et d'une didactique de la recherche d'information sur Internet, indissociable d'une pédagogie de l'enquête. Ce renforcement passe par l'étude des 
conditions et contraintes du déploiement d'une telle culture, étude à laquelle l'enquête que nous présentons a vocation à contribuer.

\section{Dispositif d'enquête}

La visée de notre dispositif d'enquête par questionnaire est de collecter des réponses volontaires nombreuses de la part d'enseignants du secondaire dans l'éducation nationale.

Nous avons demandé à tous les rectorats de France d'autoriser l'envoi via le mailing académique d'un lien pointant sur un questionnaire à renseigner sur Internet, le message étant assorti d'un court descriptif des finalités de l'enquête annoncée comme une recherche universitaire et précisant les garanties de confidentialité (en particulier vis-à-vis de l'institution de tutelle). Huit académies ont répondu à notre demande et quatre ont accepté de mettre en place la procédure. Un lien pointant sur le serveur Internet de notre unité de recherche a été envoyé dans chaque boîte de courriel des enseignants des collèges et lycées ${ }^{3}$.

L'outil d'enquête est un questionnaire « en ligne » réalisé à l'aide du logiciel $\mathrm{Le}$ Sphinx $i Q^{\circledR}$. Sur un total de 75 questions, le questionnaire comprend 19 questions fermées, 48 questions à échelles et 8 questions ouvertes.

La construction du questionnaire ne s'est pas basée sur les contenus du référentiel $\mathrm{C} 2 \mathrm{i}$ enseignants, alors que quelques items rappellent des points des référentiels B2i C2i. Une stratégie a présidée à l'enchaînement des questions : faire émerger par contraste l'usage de l'Internet par rapport aux autres usages des outils numériques. Notre enquête, contrairement à celle de Guichon (2012), ne fait pas référence directement à la sociologie des usages, ce qui l'aurait probablement rendu plus informatif. Notre approche dans le champ du didactique exige au préalable un état des lieux davantage descriptif qu'explicatif sur une large échelle.

Nous avons enregistré près de 5000 répondants et en avons conservé 4463 après élimination des doublons, des questionnaires incomplètement renseignés ou à l'évidence renseignés de manière incohérente ${ }^{4}$ par ce que nous nommons les «visiteurs curieux » qui parcourent les questions pour arriver au bout et se faire une idée globale du contenu.

\section{Résultats}

\subsection{Caractéristiques de la population}

L'échantillon de population obtenu constitué par l'ensemble des répondants validés est limité ici aux enseignants du secondaire et à l'exclusion du personnel 
d'encadrement, soit une population de 2862 enseignants. Il peut se décrire de la manière suivante :

La plus grande partie a entre 30 et 55 ans (79\%), la majorité sont des femmes $(58 \%)$, ils sont tous diplômés de l'enseignement supérieur et on compte une très bonne proportion $(62 \%)$ d'entre eux qualifiés professionnellement (Capes, Capet, agrégation, etc.). Près de la moitié a suivi une formation en informatique (48\%) et seulement $16 \%$ sont titulaire d'une certification (B2i, C2i).

Les disciplines enseignées sont pour $26 \%$ du domaine scientifique, $36 \%$ du domaine des sciences humaines et sociales, $10 \%$ du domaine artistique et de l'éducation physique et sportive, $24 \%$ de technologie et tertiaire, et enfin $4 \%$ documentalistes. Les établissements ruraux sont minoritaires (31\%) ainsi que les établissements classés en zone d'éducation prioritaire (ZEP) ou réseau d'éducation prioritaire (REP) $(9,5 \%)$.

Ce sont des personnels en plein cœur de leur carrière qui ont majoritairement répondu, bien que la proportion de «débutants » ne soit pas négligeable $(22 \%)$ alors que ceux proches de la retraite sont peu représentés $(8 \%)$. Si on rapporte ces chiffres à ceux fournis par l'INSEE pour le Ministère de l'Éducation Nationale (MEN) pour décrire la population des personnels d'enseignement du secondaire, on peut dire que notre échantillon est représentatif de l'ensemble de ces personnels.

\subsection{Quels usages de l'ordinateur et d'Internet?}

Une auto-évaluation de l'expertise sur les principaux usages des outils numériques révèle que les enseignants - toutes disciplines confondues - se sentent très à l'aise avec les traitements de texte et Internet, moyennement avec les tableurs et le traitement de l'image mais relativement gênés avec les jeux et les vidéos. On note toutefois une influence attendue de la discipline enseignée quant à l'expertise déclarée de certains outils avec les attractions suivantes : les tableurs (mieux pratiqués par les enseignants du domaine scientifique); la vidéo et la photo (arts plastiques et appliqués, musique, langues et physique chimie) ; les logiciels de présentation (technologie et tertiaire, arts plastiques, documentation, sciences de la vie et de la Terre) ; le traitement de texte (langues); Internet (documentation, langues). Les enseignants du français, du latin, de la philosophie et de l'éducation physique et sportive indiquent des maitrises en moyenne plus faibles, plus particulièrement sur les outils tableur et logiciel de présentation.

Les enseignants affirment également de manière très majoritaire que l'usage des ordinateurs a bouleversé fortement leurs pratiques professionnelles ( $44 \%$ se positionnement au niveau le plus élevé sur une échelle de 0 à 5 et si on y ajoute le niveau 4 on arrive à $67,5 \%$ ), ce qui confirme une tendance datant de plusieurs décennies comme 


\section{Caroline LADAGE, Jean RAVESTEIN}

l'écrivaient déjà en 2001 Karsenti, Savoie-Zajc, et Larose (2001). Ici encore une différence apparaît : le tableau des moyennes par discipline montre en effet des attractions avec des scores très significativement au-dessus de la moyenne pour la technologie et le tertiaire, les arts plastiques, la musique et la documentation. Nous trouvons la même tendance quant à leur rapport aux sources d'information, avec cependant des scores au-dessus de la moyenne chez des enseignants de la technologie, du tertiaire, de l'histoire et de la géographie, de la documentation et des sciences économiques et sociales, et en-dessous de la moyenne pour les enseignants de langues, de mathématiques, de philosophie et d'éducation physique et sportive.

Leur rapport à l'Internet est affirmé principalement pour poser des questions et communiquer (courriels). On note qu'une majorité utilise Internet pour préparer la classe, ainsi sur une échelle de 0 à 5 plus de la moitié se situe au niveau 4 et 5 avec respectivement $27 \%$ et $34 \%$. Les enseignants du domaine technologique et tertiaire scorent ici encore au-dessus de la moyenne, rejoints par les enseignants de langues et des sciences de la vie et de la Terre. La communication par Internet est davantage développée auprès des enseignants de langues et les documentalistes.

On remarque une certaine pauvreté technique (à l'exception des documentalistes) dans l'usage des moteurs: peu de recherche avancée, usage des guillemets et des connecteurs logiques anecdotiques (plus de $40 \%$ déclare ne jamais les utiliser). En revanche ils sont majoritaires pour l'utilisation des suggestions proposées par les moteurs. Google reste le moteur utilisé préférentiellement $(57 \%)$ comme page d'accueil à l'ouverture d'un navigateur, suivi des fournisseurs d'accès (FAI), $21 \%$. Ils se disent très habiles à récupérer, sauvegarder et réutiliser des ressources de tout format $(77 \%)$, plus particulièrement les enseignants en arts plastiques, musique et technologie. Beaucoup (82\%) se contentent de rester sur la première page de résultats que renvoie le moteur à une requête. Ils déclarent en revanche ne pas utiliser les liens sponsorisés. Les participants trouvent ce qu'ils pensent chercher assez rapidement car ils ne retournent que peu de fois sur la page de résultats avant d'avoir satisfaction. Très majoritairement ils déclarent trouver une réponse satisfaisante à leurs requêtes (80\%) et plus de $90 \%$ d'entre eux affirment ne plus pouvoir se passer de l'Internet aujourd'hui. Ils pensent à plus de $50 \%$ savoir comment les moteurs hiérarchisent les résultats des requêtes, mais en revanche, ils sont sûrs à $67 \%$ que les moteurs ignorent certains résultats qui pourraient pourtant leur être utile et que les liens proposés ne sont pas «vérifiés ». Cette relative méfiance se traduit par un certain croisement des sources ; majoritairement ( $81 \%$ ) ils cliquent sur plusieurs liens pour compléter une réponse obtenue en première instance.

Ils se montrent très peu actifs sur le Web 2.0. En effet seulement $18 \%$ ont un blogue ou un site personnel et ils sont $55 \%$ à ne jamais participer à des forums, ou 
très rarement (37\%). Ils ne fréquentent pas davantage les listes de discussions (29\% ; à l'exception des enseignants en technologie et en documentation qui y souscrivent respectivement de manière significative et très significative plus que leurs collègues), par contre une proportion non négligeable a un compte sur un réseau social (52\% ; ici ce sont les linguistes qui en font un usage le plus poussé), mais ils sont très majoritaires, à ne pas aller sur Internet pour « se faire des amis » $(74 \%) \ldots$

On note une spécialisation sans surprises des outils en fonction des disciplines enseignées à laquelle échappe toutefois Internet et le traitement de texte qui paraissent, à tort ou à raison, plus « universels ». Si on fait l'hypothèse que c'est à tort, ces déclarations d'une bonne maitrise peuvent, comme le remarque Ladage (2008), s'ériger en obstacle à toute velléité de formation à ces instruments : «je crois savoir, il n'y a donc rien à apprendre ! »

\subsection{Quelles TIC à l'école?}

Une forte majorité toutes disciplines confondues (75\%) pense que l'usage de l'Internet a changé le rapport des élèves au savoir. Dans l'exercice de leur métier, l'usage de l'Internet semble diviser la population car les réponses sont fortement contrastées. En effet, alors que $61 \%$ proposent de faire travailler les élèves avec l'Internet, les autres y semblent rétifs et alors que $51 \%$ utilisent souvent des sites de ressources pédagogiques, les autres prétendent ne jamais ou rarement les fréquenter. Le clivage reste vif quand on demande quels rapports l'Internet pourrait modifier entre eux et les élèves : $64 \%$ ne souhaitent pas communiquer directement avec les élèves par Internet alors que les autres y sont favorables. Près de la moitié de la population affirme fréquemment utiliser l'information en provenance d'Internet (61\%) pour la préparation de leur classe, contre $20 \%$ moyennement et $19 \%$ peu ou pas.

Cette évidente non congruence des opinions rejoint les résultats de Ratinaud (2003) obtenus avec une méthode qualitative (analyse de discours d'enseignants à propos de l'usage d'Internet dans le cadre professionnel). Il constate que «les discours des enseignants interrogés sur le thème d'Internet font effectivement apparaître des formes non-congruentes. On retrouve ces formulations sous leurs deux aspects (positif et négatif) ». Une vérification sur l'incidence disciplinaire révèle dans notre enquête une relation significative, voire très significative mais de façon peu systématique, que ça soit du point de vue de la discipline ou du type de clivage remarqué. On note ainsi pour le premier clivage, que les enseignants de mathématiques, de physique / chimie et d'éducation physique et sportive, déclarent moins volontiers inviter leurs élèves à travailler avec Internet, alors que c'est le contraire dans le domaine de la technologie, du tertiaire et de la documentation. Pour le deuxième clivage ce sont encore les mathématiciens et les enseignants d'éducation physique et sportive qui déclarent de façon très significative de ne pas ou rarement utiliser les sites de res- 


\section{Caroline LADAGE, Jean RAVESTEIN}

sources pédagogiques, contrairement aux documentalistes et aux enseignants de musique qui déclarent au contraire y avoir recours de manière très significative. Le clivage sur le recours à l'information disponible sur Internet pour la préparation de la classe est marqué positivement par les enseignants d'arts plastiques et appliqués, des sciences de la vie et de la Terre et de ceux de technologie, à l'inverse, il l'est négativement par ceux du français, du latin, des mathématiques et de l'éducation physique et sportive. Enfin, le quatrième clivage remarqué, portant sur les régulations individuelles des apprentissages par Internet, ne subit aucune influence disciplinaire. On constate qu'aucun profil marqué ne ressort de ces clivages, à l'exception des enseignants de mathématiques et d'éducation physique et sportive, qui basculent pour trois clivages dans une dominante négative.

Du point de vue des besoins en formation ils sont plus de $18 \%$ à affirmer qu'il n'est pas nécessaire de recevoir une formation à l'usage des moteurs de recherche pour exercer aujourd'hui leur métier, une bonne part est indécise (31\%) alors que les $51 \%$ restants la jugent indispensable. Les positions extrêmes sont marquées ici de façon négative pour les enseignants de mathématiques et de physique / chimie, qui affirment de façon très significative de ne «pas du tout » (pour les premiers) ou «plutôt pas » avoir besoin de ce type de formation ; elles sont marquées de façon positive par les enseignants d'histoire / géographie et les documentalistes pour qui cette formation est de façon très significative tout à fait nécessaire.

Une série de questions concernant l'usage de l'encyclopédie en ligne Wikipédia permet de dégager les pratiques suivantes : beaucoup l'utilisent souvent (61\%) et s'en disent satisfaits (84\%). En revanche, ils n'y participent pas (3\% seulement) et sont très majoritaires à ne pas la conseiller, ou rarement, à leurs élèves (73\%). Ils ont une attitude «consumériste » dans l'usage de l'encyclopédie : $9 \%$ seulement suivent régulièrement les mises à jour avec l'onglet «historique »; et $67 \%$ ne vérifient pas ou peu qui sont les contributeurs à un article. En revanche une minorité «fait confiance » aux contenus ( $28 \%$ ), alors que les autres restent très méfiants. Ce sont des visiteurs curieux (43\% la parcourent «comme on feuillette un livre»), beaucoup l'utilisent comme moyen de confirmation (57\%). L'analyse des relations entre les champs disciplinaires et les opinions déclarées sur Wikipédia ne permet pas de dégager de profils marqués.

On décèle donc ici un ensemble de pratiques qui montre un certain pragmatisme (Wikipédia devient incontournable) qui s'accompagne d'une mise à distance (surtout pour les élèves). Des études récentes le confirment en montrant de surcroit la vivacité de cette question dans la sphère scolaire (Garfinkel, 2008 ; Lim, 2009 ; Harouni, 2009 ; Rinne, 2009 ; Head et Eisenberg, 2010). D'autres auteurs s'engagent davantage en mesurant tout l'intérêt de l'utiliser dans un cadre pédagogique (Bruillard, 2007 ; 
Dioni, 2008 ; Vandendorpe, 2008 ; Ladage et Ravestein, 2012), alors que les établissements continuent de promouvoir l'usage des encyclopédies «officielles » accessibles gratuitement via leurs Espaces Numériques de Travail (ENT).

\subsection{Hommes, femmes, des rapports aux TIC différents}

Pour mieux comprendre le phénomène de division de la population que nous venons de citer pour un nombre non négligeable de comportements, nous avons recherché la présence d'autres variables déterminantes que la discipline enseignée pour laquelle les relations observées ne permettent pas clairement de dégager des tendances. Ni le niveau de diplôme, ni les formations en informatique ou la possession d'un $\mathrm{C} 2 \mathrm{i}$ ne sont déterminants. Par contre un certain nombre de traits marquants apparaissent avec les variables sexe et âge.

La variable sexe marque en effet depuis les débuts de l'informatique des stéréotypes concernant les usages et les représentations de l'informatique (Le Douarin, 2004 ; Drot-Delange, 2011), l'image de la femme devant l'ordinateur est dévalorisée, l'informatique apparaissant socialement comme une affaire d'homme, alors que dès le lycée les jeunes filles ne souscrivent pas aux stéréotypes selon lesquels elles seraient moins habiles à utiliser l'ordinateur (Baron, Drot-Delange, Khaneboubi et Sedouka, 2010).

Dans notre enquête sur les 2862 des participants du secondaire comportant $58 \%$ de femmes et $42 \%$ d'hommes, nous observons qu'un certain nombre de comportements déclarés diffèrent de façon significative entre hommes et femmes ${ }^{4}$. Un premier exemple concerne l'utilisation d'Internet dans la préparation de la classe, où, comme le montre la figure 1 ci-après, les hommes déclarent de façon significative plus souvent que les femmes, ne jamais utiliser Internet dans la préparation de la classe (correspondant à 0 sur l'échelle des réponses). Ils sont également en dessous de l'effectif théorique attendu (noté «Eth» dans le tableau ${ }^{5}$ ) dans la modalité de réponse correspondant au niveau de recours à Internet le plus élevé (5 sur l'échelle des réponses). Ceci confirme la tendance observée d'une minoration dans la représentation que les hommes ont quant à leur utilisation d'Internet pour la préparation de la classe. 
Caroline LADAGE, Jean RAVESTEIN

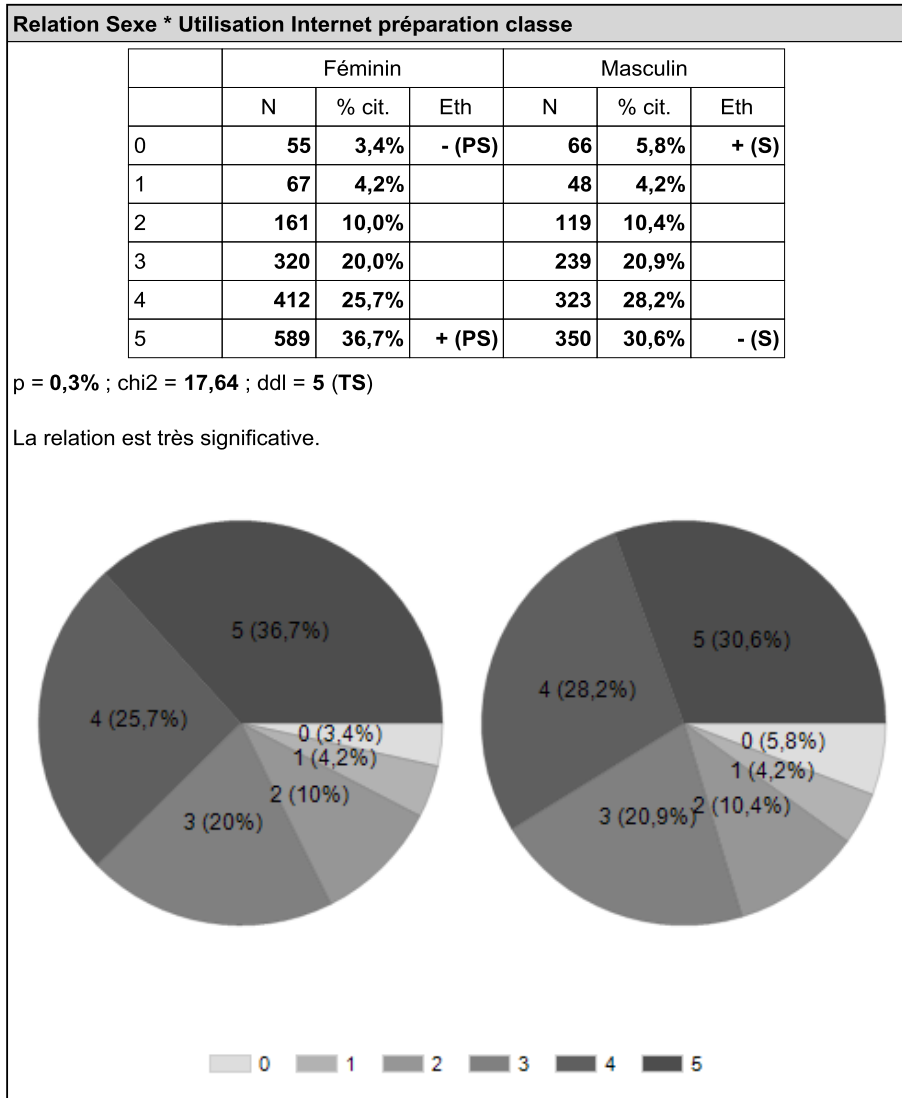

Figure 1 - Sexe / Utilisation de l'Internet pour la préparation de la classe

Le comportement des femmes et des hommes diffère encore dans la création de sites personnels et de blogues (figure 2), pratiques en ligne que les femmes déclarent bien moins souvent que les hommes, et cela de façon très significative. 


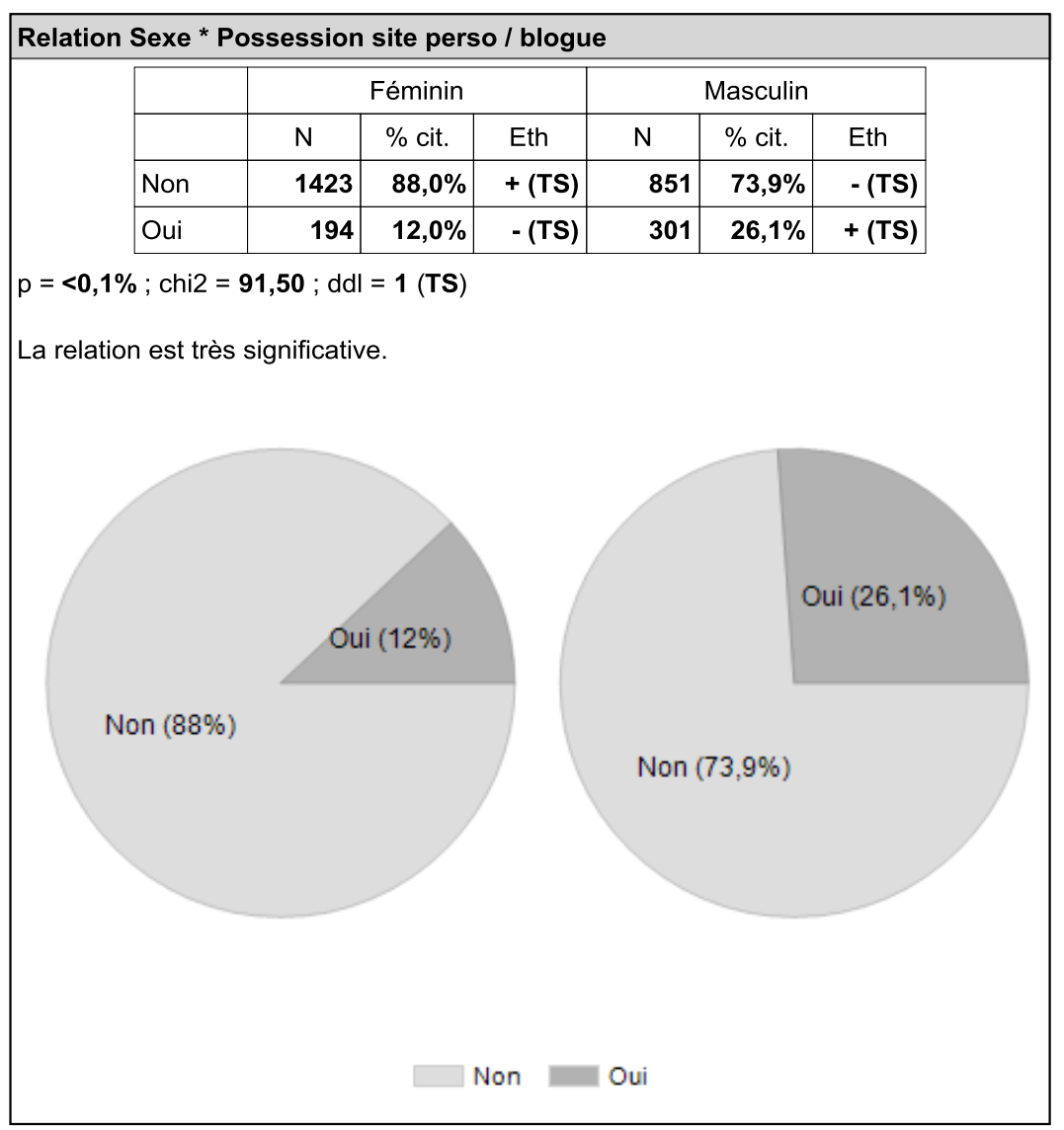

Figure 2 • Sexe / Possession d'un blogue ou d'un site Internet personnel

Une autre distinction apparaît dans la prise de parole sur les forums, que la figure 3 fait apparaître. Les comportements déclarés des hommes et des femmes diffèrent là encore de façon significative, voire très significative, les hommes étant des intervenants beaucoup plus actifs que les femmes. Ce résultat ne peut pas être étendu aux réseaux sociaux en général où on observe des comportements certes différents mais pas seulement d'un point de vue quantitatif (Lebrun et Lacelle, 2012). Notons cependant que, si les effectifs de femmes se situent en règle générale au-dessous de l'effectif théorique (correspondant à l'indépendance), il n'en est pas ainsi pour la tranche d'âge des moins de 32 ans (voir annexe ${ }^{6} 1$ ). 


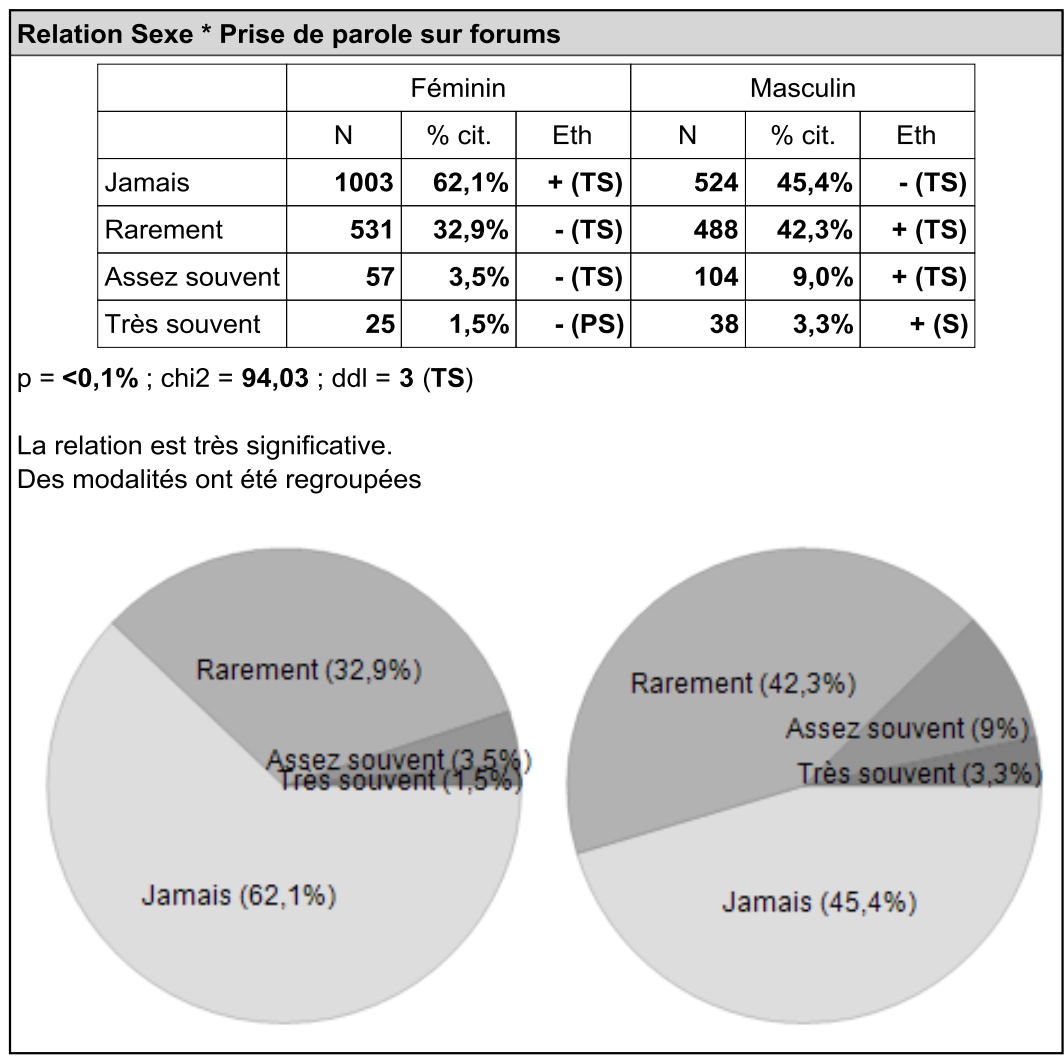

Figure $3 \cdot$ Sexe / Prise de parole sur des forums

Si l'on observe les usages des TIC de façon plus générale, ces différences de comportement entre femmes et hommes sont confirmées par les déclarations relatives au degré d'expertise sur une échelle allant de 1 (faible) à 6 (fort) en ce qui concerne les tableurs, les logiciels de présentation, les photos, les vidéos et les jeux : pour chacun de ces outils, une différence très significative est observée (annexe 2). On trouve cette différence très significative entre déclarations des hommes et des femmes d'une part dans les valeurs élevées des échelles (5 et 6), où les hommes se déclarent davantage experts alors que les femmes y sont sous-représentées, d'autre part dans les valeurs faibles de l'échelle (1 et 2), que les femmes choisissent davantage et où les hommes sont sous-représentés. Ces relations s'observent quelle que soit la tranche d'âge, à l'exception de la maîtrise de la vidéo et des photos où la part de la population se situant dans le premier tiers des classes d'âge est de manière très significative surreprésentée du côté de l'expertise avancée. Le traitement de texte fait figure à part (annexe 3): bien que la différence des comportements déclarés entre hommes et 
femmes y soit significative, elle est sensiblement moins marquée que dans les autres types de pratiques. C'est en examinant l'influence de l'âge (et ce quel que soit le sexe) que l'on voit ici se dessiner des différences. C'est ainsi que, alors que les moins de 30 ans se déclarent au niveau le plus élevé d'expertise (le niveau 6) de façon significative, les plus de 50 ans se déclarent de façon très significative dans les niveaux moins élevés de maîtrise (2, 3 et 4), tout en étant sous-représentés au niveau le plus élevé (6).

Il est enfin intéressant de noter que l'usage d'Internet - désigné de façon générale - est le seul instrument (à l'exception du traitement de texte) où la différence entre hommes et femmes est peu significative ( $\mathrm{p}>10 \%$ ). Cette absence de différence marquée ne résiste pas dès l'instant où l'on observe des fonctionnements d'outil de manière plus spécifique. À titre d'exemple, pour le fonctionnement des moteurs de recherche, le patron de la différence entre hommes et femmes ressort. Chez les femmes on note une sous-représentativité très significative de celles qui déclarent comprendre comment les moteurs de recherche hiérarchisent les résultats et une surreprésentativité équivalente de celles qui affirment n'avoir pas ou peu d'idées à ce sujet. Chez les hommes, on observe une fois de plus de façon symétrique les relations inverses (annexe 3). Si toutefois on introduit la variable âge dans l'analyse, on constate que davantage de femmes plus jeunes déclarent connaitre le fonctionnement des moteurs de recherche et rejoignent la position des hommes. Ainsi, nos résultats confirment globalement ceux de Le Douarin (2004) obtenus avec une méthode qualitative: bien que des contrastes demeurent, les déclarations des femmes sur leurs compétences dans l'usage des ordinateurs nuancent les stéréotypes «essentialistes » masculin / féminin dans leurs rapports avec les techniques, en particulier chez les plus jeunes. On pourrait nuancer la portée de nos résultats en tenant compte du fait que, concernant l'informatique et ses usages, les femmes ont tendance à se sous-estimer si on se fie à des enquêtes antérieures au WEB 2.0 (Makrakis, 1993 ; Sacks, Bellisimo et Mergendoller, 1994 ; Shashaani, 1994), mais cela semble moins vrai aujourd'hui, en particulier dans les situations où il y a un enjeu dans l'usage (Kobila, 2009).

\subsection{Que font les élèves sur Internet pour leur travail scolaire ? Regards divergents}

Cette question a fait l'objet d'une question ouverte et a recueilli un taux de réponse de $83 \%$, soit 2381 réponses (la question était formulée de la façon suivante : «Pourriez-vous dire en quelques mots ce que vous pensez que les élèves font majoritairement avec Internet dans leur travail scolaire ?»; 2097 réponses ont été retenues pour l'analyse qualitative, les 284 réponses restantes ont été écartées à cause de leur incohérence ou de leur ambiguïté). Le résultat le plus important et en même temps le plus évident de l'analyse des réponses à cette question est que les élèves font de la 
recherche d'informations, ce qui apparaît comme l'usage d'Internet le plus cité (on trouve 1457 occurrences de la forme « recherche»). Une divergence remarquable et moins attendue est cependant apparue après une analyse de contenu par repérage de catégories de réponses : le constat d'un partage de l'échantillon entre deux "pôles » majeurs, qui cette fois ne sont déterminés ni par le sexe ni par l'âge.

Le premier pôle est représenté par 48,1\% des réponses, qui suggèrent que les élèves réalisent un «évitement du travail» quand ils vont sur Internet («ils vont chercher les réponses aux devoirs de maison pour éviter de se fatiguer »; «Seuls : du "copier-coller". Essayer de trouver la correction du devoir à faire. Traduire mot à mot. Chercher le résumé du livre à lire... Au CDI, dans le cadre d'un cours, quelques recherches utiles. »). Cet évitement du travail se traduit majoritairement par l'usage de l'expression « copier-coller », qui apparaît 512 fois. Toutefois, un nouveau venu dans le paysage des usages décriés d'Internet semble être la fonction de traduction proposée par certains sites : cet usage est cité 156 fois. Si on compare ce nombre avec celui du copier-coller (512), on peut en effet voir apparaitre là ce qui semble être perçu par les enseignants comme l'un des nouveaux usages potentiellement mauvais d'Internet, ce qui est vérifié par le sens des unités de contexte élémentaires dans lesquelles le mot «traduction » est présent ( «La traduction automatique dans ma matière s'ils ont des recherches à effectuer. Ils refusent majoritairement l'effort de lire le texte directement dans la langue de départ »; "Très peu de choses : au mieux une recherche précise "guidée" par l'enseignant qui leur propose de rechercher une info ou visualiser une vidéo sur un site précis. Au pire, en langue étrangère, des traductions incertaines et maladroites à l'aide de traducteurs automatiques. »).

L'autre polarité qui se dégage est représentée par 36,9\% des réponses, qui considèrent que les élèves réalisent un «travail réel et intéressant» avec Internet («en classe : internet est un outil dont on apprend à se servir, notamment pour rechercher de l'information sur les entreprises, l'économie, le droit, etc. ; chez eux : pas beaucoup plus de curiosité qu'avec d'autres supports (magazines...) »; «approfondir ce qui a été fait en classe »). Ce type de réponse est cependant souvent nuancé par l'idée d'un apprentissage indispensable et le fait qu'un bon usage d'Internet n'est pas l'apanage de n'importe quel élève et qu'il faut être bon et savoir l'utiliser ( «s'ils sont curieux, c'est un outil formidable, s'ils sont "paresseux" et de niveau moyen, c’est [tout le contraire d'un outil] : le "copié" fait qu'ils ne réfléchissent plus personnellement et le "collé" fait qu'ils ne savent plus écrire en français. »).

Une part nettement plus faible des enseignants ayant répondu à cette question ouverte $(7,9 \%)$ y voit principalement un dispositif de divertissement («ils s'évadent»), mais on lit aussi «Ils jouent ou ils piratent, évidemment ! ! Ou alors, ils regardent des films de c... ou exposent leur mal-être d'ado à la face du monde, via MSN ou des 
blogs ! ! ! Car ça sert surtout à ça... Et on se leurre d'en faire la panacée ou je-ne-saisquoi de miraculeux. ». Enfin, alors que 5,3\% des réponses identifient un manque d'approfondissement («ils zappent et surfent sans critique », ou encore «ils impriment des pages qu'ils ne lisent pas avec le sentiment d'avoir produit un travail très approfondi »), une très faible minorité $(1,8 \%)$ décèle plutôt dans l'utilisation d'Internet par les élèves un manque d'efficacité («Dans leur travail scolaire ? Si on les laisse faire : du copier/coller! Peu d'entre eux savent rechercher, trier, filtrer, hiérarchiser l'information... et la transformer en "connaissance". Ils savent chercher et trouver, au premier niveau de l'acte »; ou encore " D'une manière générale si beaucoup d'élèves savent utiliser internet, ils ne savent pas (ou mal) l'utiliser pour approfondir leurs connaissances, il en est de même pour les adultes »). Parmi ces derniers, quelques-uns notent un manque d'apprentissage («Pour le moment leur utilisation d'internet n'est pas très efficace », ou encore «Ils apprennent. Ils apprennent que le copier-coller c'est pas efficace pour avoir de bonnes notes, et ils apprennent à reformuler, résumer, synthétiser plusieurs documents, ce qui les fait apprendre malgré eux. »). À l'échelle de l'ensemble des répondants à cette question le rôle de l'enseignant et la place d'un apprentissage ne sont cependant que très rarement évoqués. On voit se dégager deux grandes tendances contrastées de l'opinion : ceux qui pensent que l'évitement du travail est majoritaire (pôle dominant) et ceux qui y voient un travail réel intéressant.

Cette polarité d'opinion semble faire écho à ce que Zaffran (2001) montre de la temporalité des collégiens hors école : «Il s'agit pour eux d'opter soit pour un principe de renforcement de l'ethos scolaire par la contraction du temps mort et la conversion du temps libre en temps productif, soit un principe de dilapidation du temps libre ».

En revanche, ces prises de position tranchées ne rendent pas raison des multiples comportements d'usage, parfois cumulés chez le même élève, que relèvent Le Douarin et Delaunay-Téterel (2011) : "Certains lycéens “ font des coups " de temps en temps, d'autres exploitent le net pour faire face à la pression tout en ayant conscience des risques qu'ils prennent. Il n'y aurait pas non plus d'un côté des " pluriactifs " adeptes du principe de cumul qui emploieraient à bon escient le net scolaire et, de l'autre, des "fumistes ", adeptes de la culture des écrans et partisans du moindre effort ». Notons que la pratique du copier-coller, si elle est pointée de manière uniquement négative par les enseignants, c'est peut-être qu'ils n'ont pas encore pensé à la solliciter dans le cas d'études contrastives entre plusieurs sources.

On peut à ce stade s'interroger sur ce qui peut influencer de telles perceptions des usages que les élèves font de l'Internet. Si ni l'âge, ni le sexe, ni le niveau d'expertise déclaré, n'apparaissent à première vue comme des variables déterminantes, 


\section{Caroline LADAGE, Jean RAVESTEIN}

l'influence du domaine disciplinaire peut être étudiée. On découvre ainsi (sans hypothèse a priori) des différences significatives voire très significatives en fonction des domaines disciplinaires. Comme le montre le tableau 1 (voir aussi l'annexe 5), les enseignants du domaine littéraire ainsi que les documentalistes tendent à voir l'activité des élèves avec Internet de manière très significative comme un «évitement du travail », alors que les enseignants du domaine scientifique (de façon significative), ainsi que de celui de la technologie et du tertiaire (de façon très significative), considèrent davantage que les élèves font avec Internet un «travail réel et intéressant ».

\begin{tabular}{|c|c|c|c|c|c|c|}
\hline \multicolumn{7}{|c|}{ Perception Internet travail scolaire * Discipline enseignée } \\
\hline & \multicolumn{3}{|c|}{ Evitement du travail } & \multicolumn{3}{|c|}{ Travail réel intéressant } \\
\hline & $\mathrm{N}$ & $\%$ cit. & Eth & $\mathrm{N}$ & $\%$ cit. & Eth \\
\hline Domaine littéraire & 461 & $57,6 \%$ & $+(\mathrm{TS})$ & 223 & $27,8 \%$ & $-(\mathrm{TS})$ \\
\hline Domaine scientifique & 222 & $43,1 \%$ & - (PS) & 213 & $41,4 \%$ & $+(\mathbf{S})$ \\
\hline Technologie et tertiaire & 177 & $37,9 \%$ & $-(\mathrm{TS})$ & 208 & $44,5 \%$ & $+(\mathrm{TS})$ \\
\hline Domaine artistique et sport & 89 & $43,0 \%$ & & 88 & $42,5 \%$ & $+(\mathrm{PS})$ \\
\hline Documentalistes & 73 & $68,2 \%$ & $+(\mathrm{TS})$ & 26 & $24,3 \%$ & $-(\mathbf{S})$ \\
\hline \multicolumn{7}{|c|}{$p=<0,1 \% ;$ chi $2=102,87 ; d d l=16(T S)$} \\
\hline
\end{tabular}

\section{Tableau 1 - Lien entre la perception de l'Internet dans le travail scolaire et la discipline enseignée}

Il nous reste à poser la question de l'influence de l'âge à l'intérieur de chaque domaine disciplinaire. C'est là que nous découvrons que la tranche d'âge des 31 à 40 ans $^{7}$ influence de manière significative le clivage entre «évitement du travail » et «travail réel et intéressant » en faveur de cette dernière polarité, les autres tranches d'âge n'apportant aucune sur-représentativité ou sous-représentativité dans la distribution des réponses. Peut-on encore affiner ce profil ? On trouve alors que la variable déterminante à l'intérieur de cette tranche d'âge, et uniquement pour les domaines scientifiques et technologiques, est encore celle du genre : les femmes entre 31 et 40 ans s'y affirmant de façon très significative (domaine scientifique) et de façon significative (domaine technologique) pour une perception de l'Internet dans le travail scolaire comme un « travail réel et intéressant».

Cette sous-population féminine a en moyenne 9 années d'ancienneté, elle ne sort donc pas tout juste de sa formation universitaire et elle a connu les débuts de l'Internet. Que nous encourage-t-elle à penser? Elle nous montre une attitude qui mettrait en question la vision maintenant courante des femmes qui manifesteraient une certaine retenue vis-à-vis des technologies de la communication comme le mettait déjà en évidence Jouët en 2000 dans le cadre de la sociologie des usages : « de fait, si les femmes et les filles utilisent les TIC, leur appropriation paraît plus circonscrite : moindre connaissance du contenu de la technique, prédominance des usages fonction- 
nels, réticence à s'adonner pleinement au dialogue avec la machine » (Jouët, 2000). Elle confirmerait ainsi ce que Jouët suggérait en 2003, «que les relations entre les technologies de communication et le genre sont évolutives et ouvertes car elles s'inscrivent dans la dynamique de l'innovation technique et de l'innovation sociale » (Jouët, 2003).

Cette sous-population questionne aussi la vision des effets de génération dans les types d'usage et dans la nature de la perception des TIC, reconnaissant généralement dans la "génération $Y$ » (dans laquelle notre sous-population a au moins un pied : 3140 ans), une population dont les usages d'Internet iraient plus facilement de soi et ne seraient pas questionnés (on retrouve l'idée d'une alphabétisation informatique selon l'âge des enseignants par exemple chez Schumacher et Coen, 2008). Notre résultat corrobore ce que Heer et Akkari (2006) observent quand ils constatent qu'il n'y a pas de différence de fréquence d'utilisation des TIC entre les jeunes enseignants et les plus âgés qui ont bénéficié d'une formation initiale, ce qui s'expliquerait selon ces auteurs par le fait que les jeunes enseignants ont des priorités autres que l'intégration des TIC lors de leur première insertion professionnelle. Par ailleurs, ils notent que peu de programmes de formation initiale font des TIC une composante essentielle de leur curriculum : "Même si la majorité des enseignants ont eu la possibilité d'être formés par le biais de la formation continue, le fait que les novices ne se distinguent pas par un usage plus intensif des TIC devrait inciter les institutions de formation initiale à revoir la place des TIC dans les programmes » (Heer et Akkari, 2006). On retrouve ce type d'observation chez Larose, Grenon, Lenoir et Desbiens (2007), qui mettent en question la capacité des jeunes enseignants à introduire de nouvelles pratiques pédagogiques et qui constatent en s'appuyant sur différentes études internationales « qu'on ne peut pas identifier de distinction à ce propos entre les jeunes enseignants et leurs pairs plus chevronnés ».

Peut-on voir dans ce résultat quelque peu inattendu, le signe, aussi faible et fragile fût-il, d'une évolution des usages s'ancrant davantage au niveau des pratiques sociales et professionnelles, que résultant des effets de la formation des maîtres ? Il semble bien que c'est la confrontation avec le terrain qui encourage le développement de nouvelles pratiques pédagogiques. Il n'y a pas lieu alors d'attendre que les anciennes générations d'enseignants laissent la place aux jeunes pour faire évoluer le système éducatif. Comme le note Dioni (2008) on ne peut pas parler de rupture générationnelle à propos du rapport aux technologies des uns et des autres, les plus anciens ayant «majoritairement de l'outil informatique une perception beaucoup plus utilitaire et souvent ancrée dans une réalité professionnelle». La cohabitation des générations actuellement actives dans l'enseignement n'est-elle pas une situation inédite de confrontation de pratiques dont chaque génération pourrait bénéficier? Du point de vue de l'analyse didactique dans laquelle nous inscrivons notre recherche, cette situation 


\section{Caroline LADAGE, Jean RAVESTEIN}

apparaît bel et bien comme une condition favorable à la diffusion de pratiques avec Internet dans un contexte scolaire, que cela soit pour instrumenter les situations d'apprentissage ou pour former les élèves à un usage raisonné d'Internet. Il nous semble toutefois important que cette diffusion et instrumentation continuent de faire l'objet de recherches, car c'est bien par la description des pratiques telle que le préconise Sensevy (2011), que nous pouvons tendre vers plus d'intelligibilité des conduites humaines pour en reconstituer le déroulement et ainsi faire progresser leur enseignement et leur diffusion. Ces recherches dépassionneraient ainsi le débat sur le rôle des enseignants dans l'appropriation des usages didactiques de l'Internet par les élèves, débat qui «anime régulièrement plus souvent les médias que les salles des professeurs » (Dioni, 2008), et légitimeraient ces usages scolaires dans la société tout entière.

\section{Conclusion}

Nous trouvons-nous aujourd'hui dans une situation «d'abandon de poste » concernant l'enseignement de l'Internet et avec Internet ? Un tel enseignement serait-il regardé comme quasi impossible ? C'est ce que suggérait cette réponse de l'un des enquêtés : "Plus l'usage d'Internet en milieu scolaire se répand, moins les élèves l'utilisent à bon escient ; il est impossible de leur enseigner de bonnes pratiques, dans la mesure où ils pensent, au travers de leur usage privé, maîtriser la technique. » En réalité, il nous semble que de telles conclusions seraient pour le moins prématurées. Même si on peut s'accorder avec Fluckiger (2008) quand il affirme que "l'école peine à jouer un rôle dans la légitimation des pratiques culturelles numériques », à notre sens, le temps n'a pas encore suffisamment passé - au niveau de l'école comme au niveau de la société - pour que se soient créées et soient reconnues largement de «bonnes pratiques » (Ladage et Chevallard, 2011) et, plus généralement, pour que l'école dispose d'une «doctrine » élaborée, débattue, contrôlée et bien diffusée en la matière auprès de ses acteurs. Les enseignants se plaignent ainsi que les élèves iraient glaner sur Internet des «réponses toutes faites » aux questions qu'ils leur posent; mais la chose n'est-elle pas rendue possible par le fait que les questions des enseignants sont elles-mêmes «toutes faites »? À cet égard, l'Internet n'est-il pas moins une cause qu'un révélateur - un analyseur, même - du rapport scolaire traditionnel, partagé ordinairement par les élèves et leurs enseignants, à la connaissance et à l'ignorance, et donc aux « ressources » utiles pour chasser l'ignorance et construire la connaissance ? Les résultats de notre enquête peuvent être ainsi interprétés en un sens dynamique. Alors que, chez certains enseignants, le poids des pratiques scolaires anciennes rend méfiant et détermine des comportements conservateurs, une part suffisamment importante d'entre eux reconnaît l'intérêt de l'Internet et des TIC. On peut donc affirmer que le processus d'intégration de ces outils dans le travail scolaire peut continuer et franchir les étapes probablement moins vite que d'aucuns ne l'imaginent 
ou ne l'espèrent conduisant à une maturité épistémologique, didactique et culturelle qui nous semble désormais possible.

\footnotetext{
${ }^{1}$ Les TIC recouvrent un nombre très important d'outils allant du très général (Internet, par exemple) au très spécialisé (simulateurs, par exemple), cet acronyme est commode dans la communication, mais ne rend pas compte de la diversité et de l'évolutivité des pratiques liées aux outils. Les plus récents textes officiels parlent davantage d'outils numériques pour l'enseignement. Nous ne considérons donc pas les TIC comme un outil unique et figé. De la même manière parler d'Internet aujourd'hui ne peut plus se faire de façon générale devant la multiplicité des applications et la variété des usages qui en sont fait. Dans cet article nous interrogeons plus particulièrement les effets de l'accès aux ressources que l'Internet permet dans et hors du cadre scolaire que ça soit pour les élèves ou pour les enseignants.

2 Voir à ce sujet la page «Actualités" du site du ministère de l'éducation nationale http://www.education.gouv.fr/cid66812/projet-de-loi-pour-la-refondation-de-l-ecole-une-ecole-juste-pourtous-et-exigeante-pour-chacun.html.

${ }^{3}$ On peut penser que l'utilisation du mailing académique comme simple passerelle vers notre lien Internet pourrait constituer un échantillonnage invisible parmi la population des enseignants en termes de «profil » visà-vis de leur rapport privé avec l'institution, en particulier dans leur rapport avec l'autorité. Toutefois, notre courrier pointant le caractère totalement indépendant de notre enquête laisse à penser que les répondants ont participé sans pression ni influence majeures, ce qui sera confirmé par le libre ton parfois très critique des réponses aux questions ouvertes. Un envoi similaire concernait les enseignants du primaire et les personnels d'encadrement, mais nous réduirons pour ce travail notre échantillon aux seuls enseignants du secondaire.

${ }^{4}$ Ont été écarté pour des problèmes de cohérence des réponses manifestant soit de l'inattention sur des variables qualitatives (âge incohérent, durée de service incohérent, genre non renseigné...), soit des propos hors sujet en réponse aux questions ouvertes. Sans parler de biais, on peut dire que le caractère déclaratif de ce genre de méthode tempère la portée des résultats.

${ }^{5}$ Les tableaux présentés dans cet article incluent une colonne intitulée «Eth », ce qui signifie « écart à l'effectif théorique » dans le cadre du calcul du chi2 réalisé.

${ }^{6}$ Les annexes ainsi que les données associées à l'article sont accessibles via le lien permanent (Datapublication.org : Tge-adonis.fr.) http://hdl.datapublication.org/11107/p-001-017.

${ }^{7}$ Cette tranche d'âge de 31 à 40 ans est la deuxième d'un regroupement en 4 classes d'âge et comprend 600 répondants. La sous-population de sexe féminin est constituée de 366 personnes, dont 79 du domaine scientifique et 49 du domaine de la technologie et du tertiaire, correspondant à $35 \%$ de ce sous-groupe féminin (voir le tableau en annexe 4).
}

\section{BIBLIOGRAPHIE}

(Afshari, Abu Bakar, Su Luan, Abu Samah et Say Fooi, 2009)

AFSHARI M., ABU BAKAR K., SU LUAN W., ABU SAMAH B., SAY FOOI F. (2009). Factors affecting teachers' use of information and communication technology. International Journal of Instruction, 2(1), p. 76-104.

(Anderson et Reed, 1998)

ANDERSON, D. K, REED, W. M. (1998). The effects of Internet instruction, prior computer experience, and learning style on teachers' Internet attitudes and knowledge. Journal of Educational Computing Research, 19(3), p. 227-246.

(Balanskat, Blamire et Kefala, 2006)

BALANSKAT A., BLAMIRE R., KEFALA S. (2006). A review of studies of ICT impact on schools in Europe: European Schoolnet.

(Baron et Bruillard, 2002)

BARON G-L., BRUILLARD É. (Éds.) (2002). Les technologies en éducation. Perspectives de recherche et questions vives. Actes du symposium international francophone, Paris, 31 janvier et 1er février 2002, INRP, MSH et IUFM de Basse-Normandie.

(Baron, Drot-Delange, Khaneboubi et Sedouka, 2010)

BARON G.-L., DROT-DELANGE, B., KHANEBOUBI, M., SEDOOKA, A. (2010). Genre et informatique : compte rendu d'une enquête récente par questionnaire sur les opinions d'élèves 


\section{Caroline LADAGE, Jean RAVESTEIN}

de lycée. Revue de l'EPI. Disponible en ligne : http://www.epi.asso.fr/revue/articles/a1009c.htm (consulté le 19/12/12).

(Becker, 1999)

BECKER (1999). Internet use by teachers: Conditions of professional use and teacherdirected student use (Vol. 1): Center for Research on Information Technology and Organizations, the University of California, Irvine and the University of Minnesota.

(Bertrand, 2004)

BERTRAND C. (2004). Analyse des pratiques professionnelles des enseignants intégrant les TICE. Biennale de l'éducation et de la formation, $7^{\mathrm{e}}$ édition, INRP, Lyon, 14 au 17 avril 2004.

(Bielefeldt, 2005)

BIELEFELDT T. (2005). Computers and student learning: Interpreting the multivariate analysis of PISA 2000. Journal of Research on Technology in Education, 37(4), p. 339-347.

(Bielefeldt, 2006)

BIELEFELDT T. (2006). Teaching, Learning, and One-to-One Computing. National Educational Computing Conference, San Diego, July 6.

(Blondel et Tort, 2007)

BLONDEL F.-M., TORT F. (2007). Comment évaluer les compétences des lycéens en matière de tableur? Actes du colloque Environnements Informatiques pour l'Apprentissage Humain, Lausanne 2007.

(Bruillard, 2007)

BRUILLARD É. (2007). L'éducation face à Wikipédia : la rejeter ou la domestiquer ? Médialog, $\mathrm{n}^{\circ}$ 61, p. 39-45.

(Chen, 2010).

CHEN R-J. (2010). Investigating models for preservice teachers' use of technology to support student-centered learning. Computers \& Education, 55(1), p.32-42.

(Dawes, 2001)

DAWES L. (2001). What stops teachers using new technology? In M. Leask (ed.), Issues in Teaching using ICT. London : Routledge, p. 61-79.

(Dioni, 2008)

DIONI C. (2008). Métier d'élève, métier d'enseignant à l'ère numérique. Paris : INRP. Disponible en ligne: http://edutice.archivesouvertes.fr/edutice-00259563/fr/ (consulté le 19/12/12).

(Drot-Delange, 2011)

DROT-DELANGE B. (2011). Informatique et web : quelle place pour les filles ? Questions Vives, 15(8). Disponible en ligne : http://questionsvives.revues.org/809 (consulté le 19/12/12).

(Ertmer et Ottenbreit-Leftwich, 2010)

ERTMER P. A, OTTENBREIT-LEFTWICH A. T. (2010). Teacher technology change: How knowledge, confidence, beliefs, and culture intersect. Journal of Research on Technology in Education, 42(3), p. 255-284.

(Fluckiger, 2008)

FLUCKIGER C. (2008). L'école à l'épreuve de la culture numérique des élèves. Revue Française de Pédagogie, $\mathrm{N}^{\circ} 163$, p. 51-61.

(Fluckiger et Bruillard 2008)

FLUCKIGER C., BRUILLARD É. (2008). TIC: analyse de certains obstacles à la mobilisation des compétences issues des pratiques personnelles dans les activités scolaires. Colloque L'Éducation à la culture informationnelle, Université de Lille, 3, 16-18 oct. Presses de l'Enssib.

(MEN, 2001) 
FRANCE : MINISTÈRE DE L'ÉDUCATION NATIONALE (2001). Mise en place du brevet informatique et internet dans les collèges et les écoles au cours de 1'année 2000-2001. Rapport de l'Inspection générale de l'éducation nationale (IGEN).

(MEN, 2003)

FRANCE : MINISTÈRE DE L'ÉDUCATION NATIONALE (2003). Les attitudes des enseignants vis-à-vis des technologies de l'information et de la communication. Direction de l'évaluation et de la prospective (DEP). Note d'évaluation 03.04

(MEN, 2008a)

FRANCE: MINISTÈRE DE L'ÉDUCATION NATIONALE (2008). Livre vert sur l'évolution du métier d'enseignant, sous la direction de POCHARD M.

(MEN, 2008b)

FRANCE: MINISTÈRE DE L'ÉDUCATION NATIONALE (2008). Plan pour le développement du numérique à l'école. Rapport de la mission É-educ.

(Garfinkel, 2008)

GARFINKEL S. (2008). Wikipedia and the meaning of truth: Why the online encyclopedia's epistemology should worry those who care about traditional notices of accuracy. MIT Technology Review (November/December). Disponible en ligne : http://www.technologyreview.com/web/21558/ (consulté le 19/12/12).

(Gillespie, 2006)

GILLESPIE H. (2006). Unlocking learning and teaching with ICT: Identifying and overcoming barriers. London : David Fulton.

(Gueudet, Soury-Lavergne et Trouche, 2008)

GUEUDET G., SOURY-LAVERGNE S., TROUCHE L. (2008). Soutenir l'intégration des TICE : quels assistants méthodologiques pour le développement de la documentation collective des professeurs ? Exemples du SFoDEM et du dispositif Pairform@nce. Communication au colloque DIDIREM, Paris. Disponible ligne : http://www.didirem.math.jussieu.fr/colloque2008/groupe1-3.htm (consulté le 19/12/12).

(Guichon, 2012)

GUICHON N. (2012). Les usages des TIC par les lycéens - déconnexion entre usages personnels et usages scolaires. Revue des Sciences et Technologies de l'Information et de la Communication pour l'Éducation et la Formation (STICEF) 19. Disponible en ligne : http://sticef.univ-lemans.fr/num/vol2012/05-guichon/sticef_2012_guichon_05.htm (consulté le 23 mars 2013).

(Harouni, 2009)

HAROUNI H. (2009). High School Research and Critical Literacy: Social Studies With and Despite Wikipedia. Harvard Educational Review, 79(3), p. 473-494.

(Head et Eisenberg, 2010)

HEAD J., EISENBERG M. (2010) How today's college student use Wikipedia for courserelated research. First Monday, 15(3). Disponible en ligne : http://www.uic.edu/htbin/cgiwrap/bin/ojs/index.php/fm/article/view/2830/2476 (consulté le 19/12/12).

(Heer et Akkari, 2006)

HEER S., AKKARI A. (2006). Intégration des TIC par les enseignants : premiers résultats d'une enquête suisse. Revue internationale des technologies en pédagogie universitaire, 3, p. 3848. Disponible en ligne: http://edutice.archives-ouvertes.fr/edutice-00194376 (consulté le 19/12/12).

(Hennessy, Ruthven et Brindley, 2005) 


\section{Caroline LADAGE, Jean RAVESTEIN}

HENNESSY S., RUTHVEN K., BRINDLEY S. (2005). Teacher perspectives on integrating ICT into subject teaching: Commitment, constraints, caution and change. Curriculum Studies, vol. $37, \mathrm{~N}^{\circ} 2$, p. $155-192$.

(Jouët, 2003)

JOUËT J. (2003). « Technologies de communication et genre ». Réseaux, 4(120), p. 53-86. Disponible en ligne: http://www.cairn.info/revue-reseaux-2003-4-page-53.htm, DOI : 10.3917/res.120.0053 (consulté le 19/12/12).

(Karsenti, Savoie-Zajc, et Larose, 2001)

KARSENTI T., SAVOIE-ZAJC L., LAROSE F. (2001). Les futurs enseignants confrontés aux TIC : changements dans l'attitude, la motivation et les pratiques pédagogiques. Éducation et francophonie, 29(1), p. 1-29.

(Kobila, 2009)

KOBILA J. H. (2009). L'école à l'heure des nouvelles technologies : comment évoluent les compétences en informatique des enseignants? Environnements Informatiques pour l'apprentissage Humain (EIAH), Le Mans.

(Lebrun et Lacelle, 2012)

LEBRUN M., LACELLE N. (2012). Les usages linguistiques des adolescents québécois sur les médias sociaux. Alsic. Apprentissage des Langues et Systèmes d'Information et de Communication, 15(1).

(Ladage, 2008)

LADAGE C. (2008). Étude sur l'écologie et l'économie des praxéologies de la recherche d'information sur Internet. Une contribution à la didactique de l'enquête codisciplinaire. Thèse de doctorat de l'université de Provence, département des sciences de l'éducation.

(Ladage et Chevallard, 2011)

LADAGE C., CHEVALLARD Y. (2011). Enquêter avec l'Internet. Études pour une didactique de l'enquête. Éducation \& Didactique. vol. 5. N 2, p. 85-115.

(Ladage et Ravestein, 2012)

LADAGE C., RAVESTEIN J. (2012). Wikipédia à l'école, où en est-on aujourd'hui ? Journées Communication et Apprentissage Instrumentés en Réseau. Jocair, Amiens, 2012.

(Larose, Grenon, Lenoir et Desbiens, 2007)

LAROSE F., GRENON V., LENOIR Y., DESBIENS J.-F. (2007). Le rapport des futurs enseignants à l'utilisation de l'informatique pédagogique: fondements et trajectoire longitudinale. In B. Charlier, D. Peraya. Transformation des regards sur la recherche en technologie de l'éducation, p. 219-239. Bruxelles : De Boeck Université.

(Le Douarin, 2004)

LE DOUARIN L. (2004). Hommes, femmes et micro-ordinateur: une idéologie des compétences. Réseaux, 123(1), p. 149-160.

(Le Douarin et Delaunay-Téterel, 2011)

LE DOUARIN L., DELAUNAY-TETEREL H. (2011). Le net scolaire à l'épreuve du temps « libre » des lycéens. Revue Française de Socio-Économie, 8(2), p. 103-121.

(Lei, 2009)

LEI J. (2009). Digital natives as preservice teachers: What technology preparation is needed. Journal of Computing in Teacher Education, 25(3), 87-97.

(Lim, 2009)

LIM S. (2009). How and why do college students use Wikipedia? Journal of the American Society for Information Science and Technology, 60(11), p. 2189-2202. Disponible en ligne : http://sooklim.org/doc/WPJASISTLim09.pdf (consulté le 19/12/12).

(Osborne et Hennessy, 2003) 
OSBORNE J., HENNESSY S. (2003). Literature review in science education and the role of ICT: Promise, problems and future directions. London: Futurelab.

(Makrakis, 1993).

MAKRAKIS V. (1993). Gender and computing in schools in Japan: the "we can, I can't" paradox. Computers \& Education, 20(2), 191-198.

(Papi, 2012).

PAPI C. (Éd) (2012). Des référentiels à la validation des compétences numériques : questionnements et dispositifs. Questions Vive. 7(17). Disponible en ligne, http://questionsvives.revues.org/986 (consulté le 23 mars 2013).

(Pelgrum, 2001)

PELGRUM W. J. (2001). Obstacles to the integration of ICT in education: results from a worldwide educational assessment. Computers \& Education, vol. 37, p. 163-178.

(Phelps, Graham et Kerr, 2004)

PHELPS R., GRAHAM A., KERR B. (2004). Teachers \& ICT: Exploring a metacognitive approach to professional development. Australasian Journal of Educational technology, 20(1), p. 49-68.

(Ratinaud, 2003)

RATINAUD P. (2003). Enseignants du secondaire et Internet: approche d'une représentation sociale. Disponible en ligne : http://www.geirso.uqam.ca/zancien_site/jirso/Vol1_Sept03/Ratinaud_P.pdf (consulté le 19/12/12).

(Ravestein, Ladage et Johsua, 2007)

RAVESTEIN J., LADAGE C., JOHSUA S. (2007). Trouver et utiliser des informations sur Internet à l'école. Problèmes techniques et questions éthiques. Revue Française de Pédagogie, vol. 158, p. 71-84.

(Rinne, 2009)

RINNE N. (2009). Wikipedia: the educator's friend (!). TIES Conference, 15, Dec. 2009. Disponible en ligne : http://eprints.rclis.org/bitstream/10760/14002/1/Wikipedia_presentation_TIES.pdf (consulté le 19/12/12).

(Sacks, Bellisimo et Mergendoller, 1994).

SACKS C. H., BELLISIMO Y., MERGENDOLLER J. (1994). Attitudes toward computers and computer use: The issue of gender. Journal of research on Computing in education, 26, p. 256-256.

(Schoepp, 2005)

SCHOEPP K. (2005). Barriers to technology integration in a technology-rich environment. Learning and Teaching in Higher Education: Gulf Perspectives, 2(1), p. 1-24.

(Schumacher et Coen, 2008)

SCHUMACHER J.-A., COEN P. F. (2008). Les enseignants fribourgeois face aux TIC: quelle alphabétisation, quelle (s) intégration (s) ? Formation et pratiques d'enseignement en questions, 7, p. 51-71.

(Selwyn, 1999)

SELWYN N. (1999). Virtual concerns: restrictions of the Internet as a learning environment. British Journal of Educational Technology, vol. 30, p. 69-71.

Sensevy (2011)

SENSEVY G. (2011). Le sens du savoir. Éléments pour une théorie de l'action conjointe en didactique. Bruxelles : De Boeck.

(Shashaani, 1994) 
Caroline LADAGE, Jean RAVESTEIN

Shashaani, Lily. (1994). Gender-differences in computer experience and its influence on computer attitudes. Journal of Educational Computing Research, 11(4), 347-367.

(Tardif et Lessard, 1999)

TARDIF M., LESSARD C. (1999). Le travail enseignant au quotidien. Bruxelles : De Boeck.

(Vandendorpe, 2008)

VANDENDORPE C. (2008). Le phénomène Wikipédia : une utopie en marche. Le Débat 1(148), p. 17-30.

(Zaffran, 2001)

ZAFFRAN J. (2001). Le temps scolaire, le temps libre et le temps des loisirs : comment réussir au collège français en s'engageant dans la course des temps sociaux. Loisir et Société, 24(1), p. 137-149. 